\title{
The Optimal Dividend Payout Model with Terminal Values and Its Application
}

\author{
Xiankang Luo, ${ }^{1,2}$ Peimin Chen, ${ }^{1}$ and Jiangming $\mathrm{Ma}^{1}$ \\ ${ }^{1}$ School of Economic Mathematics, Southwestern University of Finance and Economics, Chengdu, Sichuan 611130, China \\ ${ }^{2}$ School of Mathematics, Yibin University, Yibin, Sichuan 644007, China \\ Correspondence should be addressed to Xiankang Luo; xkluo1978@163.com
}

Received 4 August 2017; Accepted 31 October 2017; Published 12 December 2017

Academic Editor: Quanxin Zhu

Copyright (c) 2017 Xiankang Luo et al. This is an open access article distributed under the Creative Commons Attribution License, which permits unrestricted use, distribution, and reproduction in any medium, provided the original work is properly cited.

\begin{abstract}
For some firms with large nonliquid assets, preferred shareholders can still get back a little bit of money when the firms finish disbursement of loans at the status of bankruptcy. For such a situation, to investigate the optimal dividend policy, a stochastic dynamic dividend model with nonzero terminal bankruptcy values is put forward in this paper. Moreover, an analytic solution for the optimal objective function of the discounted dividends is provided and verified. An important application of this result is that it can be employed to construct the solution for the optimal value function on the dividend problem with bailouts at bankruptcy. Further, the relationship for the solutions of these two different problems is demonstrated. In the end, some numerical examples are provided to support our theoretical results and the corresponding economic interpretations are illustrated.
\end{abstract}

\section{Introduction}

In the past decades, optimal dividend problems have been an important issue in financial and actuarial sciences. Its origin can be traced as early as the work of de Finetti [1], in which a discrete time model for optimal dividend was introduced. Recently, diffusion models for firms with controllable risk exposure and dividends payout have drawn increasing attention of researchers. We refer the readers to Jeanblanc-Picqué and Shiryaev [2], Radner and Shepp [3], Taksar and Zhou [4], Højgaard and Taksar [5, 6], Hubalek and Schachermayer [7], Cadenillas et al. [8], Paulsen [9, 10], Paulsen and Gjessing [11], Avanzi and Wong [12], Hunting and Paulsen [13], Chen et al. [14], Eisenberg [15], Vierkötter and Schmidli [16], and so forth. In all of those works, the terminal value of a company is assumed to be equal to zero when there is a status of bankruptcy, where the bankruptcy is defined as the time when the liquid assets of the company vanish. But in the real world, sometimes preferred shareholders can get some money back at bankruptcy if the value of the nonliquid assets (such as real estate or the rights to conduct business or the trade name) is large enough to pay for loans. This means that for this case the value function is not zero at the time of ruin. For such firms, how to manage the liquid assets and how to distribute dividends become a necessary problem for managers to consider. Unfortunately, there are very few results concerned with the terminal values (see, e.g., Taksar and Hunderup [17], Taksar [18], Xu and Zhou [19], and Chen and Li [20]). In addition, for firms at status of bankruptcy, sometimes they can get bailouts from governments or other firms with abundant cash flows. In such a situation, the optimal dividend policy is also an urgent problem for managers to analyze. In this paper, we put forward a dividend model with nonzero terminal values and then provide optimal dividend strategies for these two problems.

In the real financial market, capital injections are an important approach for insurance company to maintain the business when cash flow is insufficient. Recently, the optimal dividend problem with capital injections becomes hot issue in the research field of insurance. In works of Kulenko and Schimidli [21], Løkka and Zervos [22], He and Liang [23, 24], Yao et al. [25], Li and Liu [26], and so on, they assume that firms can raise capitals by issuing new shares or bonds. That implies the firms are healthy in management and financial situation and they can attract much more capitals from externals to scale up their business. In this case, the event and amounts of capital injections can be controlled by firms, 
so the capital injections can be a controllable variable in the objective function of optimal dividends (see, e.g., Løkka and Zervos [22] and $\mathrm{He}$ and Liang [23, 24]). However, nearby bankruptcy investors are in panic for prospects and firms have lots of difficulties to persuade shareholders to pay for new shares so that shareholders seldom inject capitals. In addition, at bankruptcy bailouts are mainly composed of loans or purchasing agreements from governments. For instance, in the financial crisis of 2008, the bailouts are mainly from the purchasing programs of US governments (see https://projects.propublica.org/bailout/list). Also, bailouts from governments are determined by a complicated political process, which is beyond firms' control. Therefore, in this situation it is unrealistic to treat bailouts as a controllable and internal variable for shareholders in the value function although bailouts need to be refunded in the future. In all, it is reasonable for us to view bailouts as an exogenous variable, which does not appear in objective function.

Following the classical model, we posit that the manager maximizes the expected discounted value of cumulative dividends payout. The value function is defined from shareholder's perspective; meanwhile, a residual terminal value is permitted when the firm goes bankrupt. Very closely related to the above is the problem with nonterminal bankruptcy, where a company reaches the bankruptcy state, and it does not go out of business but rather stays in this state a random amount of time and then resumes "business as usual." As in Sethi and Taksar [27], a diffusion limit description of such behavior would be via Brownian motion with delayed reflection at zero. With this setup, we then transform the optimal cumulative dividend problem to be an equivalent Hamilton-Jacobi-Bellman equation with mixed DirichletNeumann boundary condition. Further, a closed form solution and optimal dividend policy for the original problem are obtained.

The main contribution of this paper is to develop a new dividend model with terminal values or bailouts, in which bankruptcy is not terminal. A novel result obtained is the concise necessary and sufficient condition for immediate dividend events after bailouts. This result presents a guild way for regulators. To deter dividend distributions in embarrassing situation, a policy maker can set the rate of capital injection lower than the maximum dividend rate. Numerical examples are provided to support the theoretical results of the model.

The rest of this paper is organized as follows. In Section 2, we propose a mathematical model with nonzero terminal bankruptcy values for the optimal dividend problem. In Section 3, the HJB equation corresponding to this problem is derived and the detailed structure of candidate solutions is given under different situations. In Section 4, a nonterminal bankruptcy model with bailouts is presented. Then based on the results in Section 3, its smooth solution and the optimal control strategies are obtained. Meanwhile, some numerical examples are provided to verify the theoretical model and the corresponding economic interpretations are illustrated. Finally, in Section 5, we summarize our main findings and suggest a direction for future research.

\section{The Mathematical Model}

Let $(\Omega, \mathscr{F}, \mathbb{P})$ be a probability space endowed with a filtration $\left\{\mathscr{F}_{t}\right\}_{t \geq 0}$, and let $\left\{W_{t}\right\}_{t \geq 0}$ be a standard Brownian motion adapted to that filtration. To understand motivation for our diffusion control problem, one can start with the classical Cramér-Lundberg model of an insurance company. Assume that claims arrive according to a Poisson process $N_{t}$ with rate $\lambda$ and the size of $i$ th claim is $U_{i}$, where $\left\{U_{i}\right\}$ are i.i.d. with mean $\hat{\mu}$ and variance $s^{2}$. The risk process representing the liquid assets of the company, also called reserve or surplus, is governed by

$$
X_{t}=x+p t-\sum_{i=1}^{N_{t}} U_{i}
$$

where $p$ is the amount of premium per unit time received by the insurance company and the initial reserve $X_{0}=x$ is supposed to be $\mathscr{F}_{0}$-measurable. As in Taksar [28], this process can be approximated by a diffusion process with a constant drift $\mu=p-\lambda \widehat{\mu}$ and diffusion coefficient $\sigma=$ $\sqrt{s^{2}+\widehat{\mu}^{2}}$. Thus, in the absence of control the reserve process $X_{t}$ can be modelled as

$$
\begin{aligned}
d X_{t} & =\mu d t+\sigma d W_{t}, \\
X_{0} & =x .
\end{aligned}
$$

For an insurance company, it often considers to do reinsurance to reduce its risk and to pay out dividends to show its bright prospect. Proportional reinsurance, which we will consider, consists of paying a certain fraction of the premiums to the reinsurance company in exchange for an obligation from the latter to pick up the same fraction of each claim. If $1-a$ is the fraction of each claim picked up by the reinsurance company, then we call $a$ the risk exposure of the cedent. When $a$ is fixed, the reserve of the insurance company evolves as follows:

$$
X_{t}=x+a p t-\sum_{i=1}^{N_{t}} a U_{i}
$$

A diffusion approximation for the above process yields a Brownian motion with drift $a \mu$ and diffusion coefficient $a \sigma$, where $\mu$ and $\sigma$ are the same as before. We describe a control policy $\pi$ by a two-dimensional stochastic process $\left\{a_{\pi}(t), l_{\pi}(t)\right\}$, where $0 \leq a_{\pi}(t) \leq 1$ corresponds to the risk exposure and $0 \leq l_{\pi}(t) \leq M(M>0)$ is the restricted dividend rate paid out to the shareholders at time $t$. Consequently, the dynamics of the reserve process under this policy are given by

$$
\begin{aligned}
d X_{t}^{\pi} & =\left[\mu a_{\pi}(t)-l_{\pi}(t)\right] d t+\sigma a_{\pi}(t) d W_{t}, \\
X_{0}^{\pi} & =x .
\end{aligned}
$$

The set of all admissible policies is denoted by $\Pi$. For a given admissible policy $\pi \in \Pi$, the corresponding value function $V_{P}^{\pi}(x)$ is defined as

$$
V_{P}^{\pi}(x):=\mathbb{E}_{x}\left[\int_{0}^{\tau_{\pi}} e^{-\delta t} l_{\pi}(t) d t+e^{-\delta \tau_{\pi}} P\right],
$$


where $\tau_{\pi}=\inf \left\{t \geq 0 ; X_{t}^{\pi}=0\right\}$ is the time of bankruptcy, $\delta>0$ denotes a discount rate, and $P$ is the residual values left to shareholders when the company goes bankrupt. Generally, once bankruptcy occurs, shareholders have very few opportunities to get imbursements. However, in the case of some companies with large nonliquid assets, sometimes shareholders with preferred rights can still have a finger in the pie and obtain some returned money. So in this paper, we assume that $P \geq 0$.

For given $x$, the objective is to find

$$
V_{P}(x)=\sup _{\pi \in \Pi} V_{P}^{\pi}(x) .
$$

That is, we wish to find an admissible policy so as to maximize the expected present value of the cumulative dividend payouts and bankruptcy amounts shareholders achieve. The function defined by (7) is called the optimal value function, and the policy $\pi^{*}$, which satisfies $V_{P}(x)=V_{P}^{\pi^{*}}(x)$, is termed the optimal policy.

The following gives a characterization of the value function $V_{P}(x)$.

Proposition 1. The function $V_{P}(x)$ defined by (7) is concave in $[0, \infty)$.

Proof. Let $\pi_{x}$ be an admissible policy for the initial reserve $x$ and $\pi_{y}$ for the initial reserve $y$. Take $0<k<1$ and define $\pi_{z}$ by

$$
\begin{gathered}
a_{\pi_{z}}(t)=k a_{\pi_{x}}(t)+(1-k) a_{\pi_{y}}(t), \\
l_{\pi_{z}}(t)=k l_{\pi_{x}}(t)+(1-k) l_{\pi_{y}}(t) .
\end{gathered}
$$

Then, by linearity of (4), $\pi_{z}$ is an admissible policy for the initial reserve $z=k x+(1-k) y$ and $X_{t}^{\pi_{z}}=k X_{t}^{\pi_{x}}+(1-k) X_{t}^{\pi_{y}}$ with $\tau_{\pi_{z}}=\tau_{\pi_{x}} \vee \tau_{\pi_{y}}$. The linearity of (6) and (9) implies

$$
V_{P}^{\pi_{z}}(z)=k V_{P}^{\pi_{x}}(x)+(1-k) V_{P}^{\pi_{y}}(y) .
$$

For any $\varepsilon>0$, we can choose $\pi_{x}$ and $\pi_{y}$ such that

$$
\begin{aligned}
& V_{P}^{\pi_{x}}(x) \geq V_{P}(x)-\varepsilon, \\
& V_{P}^{\pi_{y}}(y) \geq V_{P}(y)-\varepsilon .
\end{aligned}
$$

As $\pi_{z}$ is suboptimal, it follows that

$$
V_{P}(z) \geq k V_{P}(x)+(1-k) V_{P}(y)-\varepsilon .
$$

From the arbitrariness of $\varepsilon$, we conclude that $V_{P}(x)$ is concave.

\section{The Hamilton-Jacobi-Bellman Equation and Its Solution}

In this section, we firstly derive the Hamilton-Jacobi-Bellman equation satisfied by $V_{P}(x)$, and then discuss how to find its analytic solutions.
For any function $\varphi(x)$, we define a differential operator $\mathscr{L}^{a, l}$ on it by

$$
\begin{array}{r}
\mathscr{L}^{a, l} \varphi(x):=\frac{1}{2} \sigma^{2} a^{2} \varphi^{\prime \prime}(x)+(\mu a-l) \varphi^{\prime}(x)-\delta \varphi(x), \\
0 \leq a \leq 1,0 \leq l \leq M .
\end{array}
$$

Proposition 2. Assume that the function $V_{P}(x)$ defined by (7) is twice continuous differential on $[0, \infty)$; then it satisfies the Hamilton-Jacobi-Bellman (HJB) equation

$$
\begin{aligned}
& \max _{a \in[0,1], l \in[0, M]}\left[\frac{1}{2} \sigma^{2} a^{2} V_{P}^{\prime \prime}(x)+(\mu a-l) V_{P}^{\prime}(x)\right. \\
& \left.-\delta V_{P}(x)+l\right]=0,
\end{aligned}
$$

with the boundary condition

$$
V_{P}(0)=P .
$$

Proof. By the similar arguments of Højgaard and Taksar [29], the HJB equation (14) can be derived. To avoid tedious repetition, here we omit it. In addition, at $x=0$, it means that a firm is at the state of bankruptcy. Under no arbitrage theorem in markets, the summation of future dividends should be equal to the residual values of the firm at bankruptcy. Thus, it follows that $V_{P}(0)=P$.

3.1. Constructing a Solution to the HJB Equation. In this section, we construct a solution $f(x)$ of (14) and (15), where for convenience $V_{P}(x)$ is replaced by $f(x)$. Let $x_{1}:=\inf \{x$ : $\left.f^{\prime}(x)=1\right\}$. Then, by concavity of $f(x)$, we have

$$
l(x)= \begin{cases}0, & x<x_{1}, \\ M, & x>x_{1} .\end{cases}
$$

Therefore, for all $x<x_{1}$, the HJB equation (14) becomes

$$
\max _{a \in[0,1]}\left[\frac{1}{2} \sigma^{2} a^{2} f^{\prime \prime}(x)+\mu a f^{\prime}(x)-\delta f(x)\right]=0 .
$$

Let $a(x)$ be the maximizer of the expression on the left-hand side of (17); then

$$
a(x)=-\frac{\mu f^{\prime}(x)}{\sigma^{2} f^{\prime \prime}(x)} .
$$

Put (18) into (17); we obtain

$$
-\frac{\mu^{2}\left[f^{\prime}(x)\right]^{2}}{2 \sigma^{2} f^{\prime \prime}(x)}-\delta f(x)=0 .
$$

A general solution of (19) with the boundary condition $f(0)=P$ is given by

$$
f(x)=C_{1}\left[x+\left(\frac{P}{C_{1}}\right)^{1 / \gamma}\right]^{\gamma},
$$


where $C_{1}$ is a free constant and $\gamma$ is given by

$$
\gamma=\frac{\delta}{\delta+\mu^{2} / 2 \sigma^{2}}
$$

This solution, however, is valid only when $a(x) \in(0,1)$. From (18) and (20), it follows that

$$
a(x)=\frac{\mu}{(1-\gamma) \sigma^{2}}\left[x+\left(\frac{P}{C_{1}}\right)^{1 / \gamma}\right] .
$$

Since $a(x)$ above is an increasing linear function of $x$, then $a(x) \leq 1$ if and only if $x \leq x_{0}$, where

$$
x_{0}=\frac{(1-\gamma) \sigma^{2}}{\mu}-\left(\frac{P}{C_{1}}\right)^{1 / \gamma} .
$$

Comparing $x_{0}$ and $x_{1}$, there are two possible cases to be considered: $x_{1} \geq x_{0}$ and $x_{1}<x_{0}$, whose necessary and sufficient conditions will be given later.

3.1.1. The Case: $x_{1} \geq x_{0}$. From (23), we notice that $x_{0}$ depends on the uncertain parameter $C_{1}$, which would lead to two different cases for $x_{0}$. So, in the following we consider two cases, $x_{0}>0$ and $x_{0} \leq 0$, and discuss them, respectively.

If $x_{0}>0$, then $a(x) \geq 1$ for $x_{0} \leq x<x_{1}$. But as $a(x) \in$ $[0,1]$, we must have $a(x)=1$ for $x \in\left[x_{0}, x_{1}\right)$. Consequently, (17) becomes

$$
\frac{1}{2} \sigma^{2} f^{\prime \prime}(x)+\mu f^{\prime}(x)-\delta f(x)=0
$$

A general solution to (24) is given by

$$
f(x)=C_{2} e^{\theta_{+}\left(x-x_{0}\right)}+C_{3} e^{\theta_{-}\left(x-x_{0}\right)}, \quad x_{0} \leq x<x_{1},
$$

where $\theta_{+}$and $\theta_{-}$are defined by

$$
\begin{aligned}
& \theta_{+}=\frac{-\mu+\sqrt{\mu^{2}+2 \delta \sigma^{2}}}{\sigma^{2}}, \\
& \theta_{-}=\frac{-\mu-\sqrt{\mu^{2}+2 \delta \sigma^{2}}}{\sigma^{2}},
\end{aligned}
$$

and $C_{2}, C_{3}$ are free constants.

The continuity of the function $f(x)$ and its derivative $f^{\prime}(x)$ at $x_{0}$ implies that

$$
\begin{aligned}
& C_{2}=C_{1} a_{1}, \\
& C_{3}=C_{1} a_{2},
\end{aligned}
$$

where

$$
\begin{aligned}
& a_{1}=\frac{\beta^{\gamma-1}\left(\gamma-\theta_{-} \beta\right)}{\theta_{+}-\theta_{-}}, \\
& a_{2}=\frac{\beta^{\gamma-1}\left(\theta_{+} \beta-\gamma\right)}{\theta_{+}-\theta_{-}},
\end{aligned}
$$

and $\beta=(1-\gamma) \sigma^{2} / \mu$.

For $x \geq x_{1}$, we have $a(x)=1$ and $l(x)=M$. Thus, $f(x)$ satisfies the following equation:

$$
\frac{1}{2} \sigma^{2} f^{\prime \prime}(x)+(\mu-M) f^{\prime}(x)-\delta f(x)+M=0 .
$$

Concavity of $f(x)$ implies that $f(x) \sim a x+b$ when $x \rightarrow \infty$ with $a, b>0$. Thereby, a solution of (29) is given by

$$
f(x)=\frac{M}{\delta}+C_{4} e^{\hat{\theta}\left(x-x_{1}\right)}, \quad x \geq x_{1}
$$

where

$$
\widehat{\theta}=\frac{-(\mu-M)-\sqrt{(\mu-M)^{2}+2 \delta \sigma^{2}}}{\sigma^{2}} .
$$

Summarizing the above, we get the structure of the solution to (14) and (15) for $x_{0}>0$

$$
\begin{aligned}
& f(x) \\
& \quad= \begin{cases}C_{1}\left[x+\left(\frac{P}{C_{1}}\right)^{1 / \gamma}\right]^{\gamma}, & 0 \leq x<x_{0}, \\
C_{1} a_{1} e^{\theta_{+}\left(x-x_{0}\right)}+C_{1} a_{2} e^{\theta_{-}\left(x-x_{0}\right)}, & x_{0} \leq x<x_{1}, \\
\frac{M}{\delta}+C_{4} e^{\hat{\theta}\left(x-x_{1}\right)}, & x \geq x_{1},\end{cases}
\end{aligned}
$$

where $C_{1}, C_{4}$, and $x_{1}$ are unknown constants and $x_{0}$ are determined by (23).

By continuity of $f^{\prime}(x)$ and $f^{\prime \prime}(x)$ at $x_{1}$, it implies that

$$
\begin{aligned}
C_{4} \widehat{\theta} & =1, \\
C_{1} a_{1} \theta_{+} e^{\theta_{+}\left(x_{1}-x_{0}\right)}+C_{1} a_{2} \theta_{-} e^{\theta_{-}\left(x_{1}-x_{0}\right)} & =1, \\
C_{1} a_{1} \theta_{+}^{2} e^{\theta_{+}\left(x_{1}-x_{0}\right)}+C_{1} a_{2} \theta_{-}^{2} e^{\theta_{-}\left(x_{1}-x_{0}\right)} & =\widehat{\theta},
\end{aligned}
$$

where we have used (33) in (35).

In view of (33), we get

$$
C_{4}=\frac{1}{\widehat{\theta}}
$$

Dividing (35) by (34), we eliminate $C_{1}$ and solve for $x_{1}$, thereby obtaining

$$
x_{1}=x_{0}+\frac{1}{\theta_{+}-\theta_{-}} \ln \left(\frac{a_{2} \theta_{-}\left(\hat{\theta}-\theta_{-}\right)}{a_{1} \theta_{+}\left(\theta_{+}-\hat{\theta}\right)}\right) \text {. }
$$


To simplify this expression, let $\alpha=\sqrt{\mu^{2}+2 \delta \sigma^{2}}$. Then $\theta_{+} \theta_{-}=-2 \delta / \sigma^{2}, \gamma=2 \delta \sigma^{2} / \alpha^{2}$, and $\beta=\mu \sigma^{2} / \alpha^{2}$. This yields

$$
\begin{aligned}
\gamma-\theta_{-} \beta & =\frac{2 \delta \sigma^{2}-\mu \sigma^{2} \theta_{-}}{\alpha^{2}}=\frac{\left(\mu^{2}+2 \delta \sigma^{2}\right)+\mu \alpha}{\alpha^{2}} \\
& =\frac{\alpha^{2}+\mu \alpha}{\alpha^{2}}=1+\frac{\mu}{\alpha}, \\
a_{1} & =\frac{\sigma^{2} \beta^{\gamma-1}(1+\mu / \alpha)}{2 \alpha}=\frac{\beta^{\gamma}(\mu+\alpha)}{2 \mu} \\
& =\frac{\sigma^{2} \beta^{\gamma}}{2 \mu}\left(-\theta_{-}\right) .
\end{aligned}
$$

By the same argument as above, we have

$$
a_{2}=\frac{\sigma^{2} \beta^{\gamma}}{2 \mu}\left(-\theta_{+}\right) .
$$

Substituting (39) and (40) into (37), we get

$$
x_{1}=x_{0}+\frac{1}{\theta_{+}-\theta_{-}} \ln \left(\frac{\hat{\theta}-\theta_{-}}{\theta_{+}-\hat{\theta}}\right) .
$$

By (34), we easily obtain

$$
\begin{aligned}
C_{1} & =\left[a_{1} \theta_{+} e^{\theta_{+}\left(x_{1}-x_{0}\right)}+a_{2} \theta_{-} e^{\theta_{-}\left(x_{1}-x_{0}\right)}\right]^{-1} \\
& =\left[a_{1} \theta_{+}\left(\frac{\widehat{\theta}-\theta_{-}}{\theta_{+}-\widehat{\theta}}\right)^{\theta_{+} /\left(\theta_{+}-\theta_{-}\right)}\right. \\
& \left.+a_{2} \theta_{-}\left(\frac{\widehat{\theta}-\theta_{-}}{\theta_{+}-\widehat{\theta}}\right)^{\theta_{-} /\left(\theta_{+}-\theta_{-}\right)}\right]^{-1} \\
& =\frac{\mu \beta^{-\gamma}}{\delta}\left[\left(\frac{\hat{\theta}-\theta_{-}}{\theta_{+}-\widehat{\theta}}\right)^{\theta_{+} /\left(\theta_{+}-\theta_{-}\right)}\right. \\
& \left.+\left(\frac{\widehat{\theta}-\theta_{-}}{\theta_{+}-\hat{\theta}}\right)^{\theta_{-} /\left(\theta_{+}-\theta_{-}\right)}\right]^{-1} .
\end{aligned}
$$

Consequently, all free constants have been determined. In addition, as in Lemma 2.1 of Højgaard and Taksar [29], it shows that $x_{1} \geq x_{0}$ if and only if $M \geq \mu / 2+\delta \sigma^{2} / \mu$.

Remark 3. From (23), it easily shows that the assumption, $x_{0}>0$, is equivalent to

$$
P<C_{1} \beta^{\gamma}
$$

that is,

$$
\begin{aligned}
& P \\
& <\frac{\mu}{\delta}\left[\left(\frac{\widehat{\theta}-\theta_{-}}{\theta_{+}-\widehat{\theta}}\right)^{\theta_{+} /\left(\theta_{+}-\theta_{-}\right)}+\left(\frac{\widehat{\theta}-\theta_{-}}{\theta_{+}-\hat{\theta}}\right)^{\theta_{-} /\left(\theta_{+}-\theta_{-}\right)}\right]^{-1} .
\end{aligned}
$$

On the other hand, for the case of $x_{0} \leq 0$, namely,

$$
\geq \frac{\mu}{\delta}\left[\left(\frac{\widehat{\theta}-\theta_{-}}{\theta_{+}-\widehat{\theta}}\right)^{\theta_{+} /\left(\theta_{+}-\theta_{-}\right)}+\left(\frac{\widehat{\theta}-\theta_{-}}{\theta_{+}-\widehat{\theta}}\right)^{\theta_{-} /\left(\theta_{+}-\theta_{-}\right)}\right]^{-1},
$$

we also need to discuss the following two cases by $x_{1}>0$ and $x_{1} \leq 0$, respectively.

If $x_{0} \leq 0$ and $x_{1}>0$, it implies that $a(x) \equiv 1$ for all $x>0$. Then, the HJB equation (14) becomes

$$
\begin{aligned}
\frac{1}{2} \sigma^{2} f^{\prime \prime}(x)+\mu f^{\prime}(x)-\delta f(x) & =0, \\
0 & \leq x<x_{1}, \\
\frac{1}{2} \sigma^{2} f^{\prime \prime}(x)+(\mu-M) f^{\prime}(x)-\delta f(x)+M & =0, \\
& x \geq x_{1} .
\end{aligned}
$$

Using the similar argument as above, we obtain a general solution of (46) as follows:

$$
f(x)= \begin{cases}C_{2} e^{\theta_{+} x}+C_{3} e^{\theta_{-} x}, & 0 \leq x<x_{1}, \\ \frac{M}{\delta}+C_{4} e^{\hat{\theta}\left(x-x_{1}\right)}, & x \geq x_{1},\end{cases}
$$

where $\theta_{ \pm}$is given by (26), $\hat{\theta}$ by (31), and $C_{2}, C_{3}, C_{4}$, and $x_{1}$ need to be determined.

The continuity of $f^{\prime}(x)$ and $f^{\prime \prime}$ at $x_{1}$, together with $f(0)=$ $P$, implies that

$$
\begin{aligned}
C_{2}+C_{3} & =P, \\
C_{4} \widehat{\theta} & =1, \\
C_{2} \theta_{+} e^{\theta_{+} x_{1}}+C_{3} \theta_{-} e^{\theta_{-} x_{1}} & =1, \\
C_{2} \theta_{+}^{2} e^{\theta_{+} x_{1}}+C_{3} \theta_{-}^{2} e^{\theta_{-} x_{1}} & =\widehat{\theta} .
\end{aligned}
$$

By (49), we get

$$
C_{4}=\frac{1}{\hat{\theta}} .
$$

Combining (50) and (51), for given $x_{1}$ we can solve for $C_{2}$ and $\mathrm{C}_{3}$

$$
\begin{aligned}
& C_{2}=\frac{\widehat{\theta}-\theta_{-}}{\theta_{+}\left(\theta_{+}-\theta_{-}\right)} e^{-\theta_{+} x_{1}}, \\
& C_{3}=\frac{\theta_{+}-\widehat{\theta}}{\theta_{-}\left(\theta_{+}-\theta_{-}\right)} e^{-\theta_{-} x_{1}} .
\end{aligned}
$$

Put (53) into (48); we obtain the following equation, where $x_{1}$ must satisfy

$$
\frac{\widehat{\theta}-\theta_{-}}{\theta_{+}\left(\theta_{+}-\theta_{-}\right)} e^{-\theta_{+} x_{1}}+\frac{\theta_{+}-\hat{\theta}}{\theta_{-}\left(\theta_{+}-\theta_{-}\right)} e^{-\theta_{-} x_{1}}=P .
$$


By continuity of $f(x)$ at $x_{1},(52)$ and (53), it follows that

$$
\begin{aligned}
C_{2} e^{\theta_{+} x_{1}}+C_{3} e^{\theta_{-} x_{1}} & =\frac{\widehat{\theta}-\theta_{-}}{\theta_{+}\left(\theta_{+}-\theta_{-}\right)}+\frac{\theta_{+}-\hat{\theta}}{\theta_{-}\left(\theta_{+}-\theta_{-}\right)} \\
& =\frac{M}{\delta}+\frac{1}{\hat{\theta}} .
\end{aligned}
$$

Remark 4. Let $g(x)=\left(\left(\widehat{\theta}-\theta_{-}\right) / \theta_{+}\left(\theta_{+}-\theta_{-}\right)\right) e^{-\theta_{+} x_{1}}+\left(\left(\theta_{+}-\right.\right.$ $\left.\widehat{\theta}) / \theta_{-}\left(\theta_{+}-\theta_{-}\right)\right) e^{-\theta_{-} x_{1}}$. Under the condition of $M \geq \mu / 2+$ $\delta \sigma^{2} / \mu$, it is easily verified that $g^{\prime}(x)<0$ on $[0, \infty)$. Thus, $\max _{x \in[0, \infty)} g(x)=g(0)=\left(\widehat{\theta}-\theta_{-}\right) / \theta_{+}\left(\theta_{+}-\theta_{-}\right)+\left(\theta_{+}-\widehat{\theta}\right) / \theta_{-}\left(\theta_{+}-\right.$ $\left.\theta_{-}\right)=M / \delta+1 / \widehat{\theta}$ owing to (55). Suppose that $P$ satisfies

$$
\begin{aligned}
& \frac{\mu}{\delta}\left[\left(\frac{\hat{\theta}-\theta_{-}}{\theta_{+}-\hat{\theta}}\right)^{\theta_{+} /\left(\theta_{+}-\theta_{-}\right)}+\left(\frac{\hat{\theta}-\theta_{-}}{\theta_{+}-\hat{\theta}}\right)^{\theta_{-} /\left(\theta_{+}-\theta_{-}\right)}\right]^{-1} \leq P \\
& <\frac{M}{\delta}+\frac{1}{\widehat{\theta}}
\end{aligned}
$$

then by intermediate value theorem, it shows that (54) has a unique positive root $x_{1}$. Thereby, the values of $C_{2}$ and $C_{3}$ can be determined certainly.

Secondly, if $x_{0} \leq 0$ and $x_{1} \leq 0$, that is,

$$
P \geq \frac{M}{\delta}+\frac{1}{\widehat{\theta}}
$$

we get (29) and find as before a solution

$$
f(x)=\frac{M}{\delta}+C_{4} e^{\hat{\theta} x}, \quad x \geq 0
$$

where $C_{4}=P-M / \delta$.

Summarizing the above, in the case of $M \geq \mu / 2+\delta \sigma^{2} / \mu$, the solutions of (14) and (15) are presented by the following theorem.

Theorem 5. Assume that $M \geq \mu / 2+\delta \sigma^{2} / \mu$. Let $\theta_{+}, \theta_{-}, \widehat{\theta}$, and $\gamma$ be defined as above.

If (44) holds, then the solution to (14) and (15) is given by

$$
f(x)
$$

$$
= \begin{cases}C_{1}\left[x+\left(\frac{P}{C_{1}}\right)^{1 / \gamma}\right]^{\gamma}, & 0 \leq x<x_{0}, \\ C_{1} a_{1} e^{\theta_{+}\left(x-x_{0}\right)}+C_{1} a_{2} e^{\theta_{-}\left(x-x_{0}\right)}, & x_{0} \leq x<x_{1}, \\ \frac{M}{\delta}+\frac{1}{\hat{\theta}} e^{\hat{\theta}\left(x-x_{1}\right)}, & x \geq x_{1},\end{cases}
$$

where $C_{1}$ is given by (42), $x_{0}$ by (23), and $x_{1}$ by (41).

If $(56)$ is true, then

$$
f(x)= \begin{cases}C_{2} e^{\theta_{+} x}+\left(P-C_{2}\right) e^{\theta_{-} x}, & 0 \leq x<x_{1}, \\ \frac{M}{\delta}+\frac{1}{\hat{\theta}} e^{\hat{\theta}\left(x-x_{1}\right)}, & x \geq x_{1},\end{cases}
$$

where $C_{2}$ is given by (53) and $x_{1}$ is determined by (54).
If (57) is true, then

$$
f(x)=\frac{M}{\delta}+\left(P-\frac{M}{\delta}\right) e^{\hat{\theta} x}, \quad x \geq 0 .
$$

Moreover, in any of the cases described above, $f(x)$ is a concave solution of (14) and (15).

Proof. From the construction above, it is easily to verify that the expressions (59)-(61) are solutions to (14) and (15). In addition, the proof on concavity of $f(x)$ is similar to that of Theorem 2.1 in Højgaard and Taksar [29], so we omit it here.

3.1.2. The Case: $x_{1}<x_{0}$. Now we turn to the case $x_{1}<x_{0}$, which is equivalent to $M<\mu / 2+\delta \sigma^{2} / \mu$. From Højgaard and Taksar [29], it shows that there exists only one "switching point" $x_{1}$ because of $x_{0}=\infty$. Therefore, the solution of (14) and (15) depends on whether $x_{1}>0$ or $x_{1} \leq 0$.

If $x_{1}>0$, for $x<x_{1}$, we obtain (17) and find as before a solution as follows:

$$
f(x)=C_{1}\left[x+\left(\frac{P}{C_{1}}\right)^{1 / \gamma}\right]^{\gamma},
$$

where $\gamma$ is given by (21) and $C_{1}$ is a free constant.

For $x \geq x_{1}$, we have $l(x)=M$. Thus, the HJB equation (14) becomes

$$
\begin{aligned}
& \max _{a \in[0,1]}\left[\frac{1}{2} \sigma^{2} a^{2} f^{\prime \prime}(x)+(\mu a-M) f^{\prime}(x)-\delta f(x)\right. \\
& +M]=0 .
\end{aligned}
$$

Let $f^{*}(x)$ be a solution of the following homogeneous equation:

$$
\begin{aligned}
& \max _{a \in[0,1]}\left[\frac{1}{2} \sigma^{2} a^{2} f^{\prime \prime}(x)+(\mu a-M) f^{\prime}(x)-\delta f(x)\right] \\
& \quad=0
\end{aligned}
$$

Then, a solution of (63) can be expressed by

$$
f(x)=f^{*}(x)+\frac{M}{\delta} .
$$

Differentiating (64) with respect to $a$ yields the maximizer

$$
a(x)=-\frac{\mu f^{\prime}(x)}{\sigma^{2} f^{\prime \prime}(x)} .
$$

Substituting (66) into (64), we obtain

$$
-\frac{\mu^{2}\left[f^{\prime}(x)\right]^{2}}{2 \sigma^{2} f^{\prime \prime}(x)}-M f^{\prime}(x)-\delta f(x)=0 .
$$


A general solution of (67) can be given by

$$
f^{*}(x)=C_{2} e^{\rho\left(x-x_{1}\right)}, \quad x \geq x_{1},
$$

where $C_{2}\left(C_{2} \neq 0\right)$ and $\rho$ are free constants.

Input (68) into (67); we obtain

$$
C_{2}\left(-\frac{\mu^{2}}{2 \sigma^{2}}-M \rho-\delta\right) e^{\rho\left(x-x_{1}\right)}=0 .
$$

Then, it is easy to derive that

$$
\rho=-\frac{\delta}{M \gamma},
$$

where $\gamma$ is defined by (21).

Thus, combining (65), (68), and (70), a solution of (63) is given by

$$
f(x)=C_{2} e^{-(\delta / M \gamma)\left(x-x_{1}\right)}+\frac{M}{\delta}, \quad x \geq x_{1} .
$$

Consequently, for the case of $x_{1}>0$, the following solution to (14) and (15) is suggested

$$
f(x)= \begin{cases}C_{1}\left[x+\left(\frac{P}{C_{1}}\right)^{1 / \gamma}\right]_{M}^{\gamma}, & 0 \leq x<x_{1}, \\ C_{2} e^{-(\delta / M \gamma)\left(x-x_{1}\right)}+\frac{M}{\delta}, & x \geq x_{1},\end{cases}
$$

where $C_{1}, C_{2}$, and $x_{1}$ need to be specified. tions:

Making "smooth fit" at $x_{1}$, we have the following equa-

$$
\begin{aligned}
-C_{2} \frac{\delta}{M \gamma} & =1, \\
C_{1} \gamma\left[x_{1}+\left(\frac{P}{C_{1}}\right)^{1 / \gamma}\right]^{\gamma-1} & =1, \\
C_{1} \gamma(\gamma-1)\left[x_{1}+\left(\frac{P}{C_{1}}\right)^{1 / \gamma}\right]^{\gamma-2} & =-\frac{\delta}{M \gamma},
\end{aligned}
$$

where we have used (73) in (75).

From (73), we obtain

$$
C_{2}=-\frac{M \gamma}{\delta}
$$

Dividing (75) by (74) yields that

$$
x_{1}+\left(\frac{P}{C_{1}}\right)^{1 / \gamma}=\frac{M \gamma(1-\gamma)}{\delta} .
$$

Put (77) into (74); we get

$$
C_{1}=\frac{1}{\gamma}\left[\frac{M \gamma(1-\gamma)}{\delta}\right]^{1-\gamma} .
$$

Combining (77) with (78), it follows that

$$
x_{1}=\frac{M \gamma(1-\gamma)}{\delta}\left[1-\left(\frac{\delta P}{M(1-\gamma)}\right)^{1 / \gamma}\right] \text {. }
$$

In addition, by (79), it shows that $x_{1}>0$ is equivalent to

$$
P<\frac{M(1-\gamma)}{\delta} .
$$

On the other hand, if $x_{1} \leq 0$, that is,

$$
P \geq \frac{M(1-\gamma)}{\delta},
$$

we get (63) and find as above a solution as follows:

$$
f(x)=C_{2} e^{-(\delta / M \gamma) x}+\frac{M}{\delta}, \quad x \geq 0
$$

By the boundary condition $f(0)=P$, we have

$$
f(x)=\left(P-\frac{M}{\delta}\right) e^{-(\delta / M \gamma) x}+\frac{M}{\delta}, \quad x \geq 0 .
$$

Since $a(x)<1$ for all $x \in[0, \infty)$, the following theorem follows easily from construction.

Theorem 6. Assume that $M<\mu / 2+\delta \sigma^{2} / \mu$. Let $\gamma$ be defined by (21).

If (80) is true, then the solution to (14) and (15) is given by

$$
f(x)= \begin{cases}C_{1}\left[x+\left(\frac{P}{C_{1}}\right)^{1 / \gamma}\right]^{\gamma}, & 0 \leq x<x_{1}, \\ \frac{M}{\delta}\left(1-\gamma e^{-(\delta / M \gamma)\left(x-x_{1}\right)}\right), & x \geq x_{1},\end{cases}
$$

where $C_{1}$ is given by (78) and $x_{1}$ by (79).

On the other hand, if (81) holds, then

$$
f(x)=\left(P-\frac{M}{\delta}\right) e^{-(\delta / M \gamma) x}+\frac{M}{\delta}, \quad x \geq 0 .
$$

Moreover, the functions $f(x)$ defined as above are concave solutions of (14) and (15).

3.2. Optimal Policy. Based on sections above, in the following, we present our optimal policy. The admissible policy $\pi^{*}$ for all $t<\tau_{\pi^{*}}$ is defined as follows.

Case $1\left(M \geq \mu / 2+\delta \sigma^{2} / \mu\right)$. (1) If (44) holds, then

$$
\begin{aligned}
& a_{\pi^{*}}(t):=a\left(X_{t}^{\pi^{*}}\right) \\
& = \begin{cases}\frac{\mu}{(1-\gamma) \sigma^{2}}\left[X_{t}^{\pi^{*}}+\left(\frac{P}{C_{1}}\right)^{1 / \gamma}\right], & 0 \leq X_{t}^{\pi^{*}}<x_{0}, \\
1, & X_{t}^{\pi^{*}} \geq x_{0},\end{cases} \\
& l_{\pi^{*}}(t):=l\left(X_{t}^{\pi^{*}}\right)= \begin{cases}0, & 0 \leq X_{t}^{\pi^{*}}<x_{1}, \\
M, & X_{t}^{\pi^{*}} \geq x_{1},\end{cases}
\end{aligned}
$$

where $x_{0}$ is given by (23) and $x_{1}$ by (41). 
(2) If (56) is true, then

$$
\begin{aligned}
& a_{\pi^{*}}(t):=a\left(X_{t}^{\pi^{*}}\right)=1, \quad X_{t}^{\pi^{*}} \geq 0, \\
& l_{\pi^{*}}(t):=l\left(X_{t}^{\pi^{*}}\right)= \begin{cases}0, & 0 \leq X_{t}^{\pi^{*}}<x_{1}, \\
M, & X_{t}^{\pi^{*}} \geq x_{1},\end{cases}
\end{aligned}
$$

where $x_{1}$ is given by (54).

(3) If (57) holds, then

$$
\begin{gathered}
a_{\pi^{*}}(t):=a\left(X_{t}^{\pi^{*}}\right)=1, \quad X_{t}^{\pi^{*}} \geq 0, \\
l_{\pi^{*}}(t):=l\left(X_{t}^{\pi^{*}}\right)=M, \quad X_{t}^{\pi^{*}} \geq 0 .
\end{gathered}
$$

Case $2\left(M<\mu / 2+\delta \sigma^{2} / \mu\right)$. (1) If (80) is true, then

$$
\begin{aligned}
& a_{\pi^{*}}(t):=a\left(X_{t}^{\pi^{*}}\right) \\
& = \begin{cases}\frac{\mu}{(1-\gamma) \sigma^{2}}\left[X_{t}^{\pi^{*}}+\left(\frac{P}{C_{1}}\right)^{1 / \gamma}\right], & 0 \leq X_{t}^{\pi^{*}}<x_{1}, \\
\frac{\mu M \gamma}{\delta \sigma^{2}}, & X_{t}^{\pi^{*}} \geq x_{1},\end{cases} \\
& l_{\pi^{*}}(t):=l\left(X_{t}^{\pi^{*}}\right)= \begin{cases}0, & 0 \leq X_{t}^{\pi^{*}}<x_{1}, \\
M, & X_{t}^{\pi^{*}} \geq x_{1},\end{cases}
\end{aligned}
$$

where $x_{1}$ is given by (79).

(2) If (81) holds, then

$$
\begin{aligned}
& a_{\pi^{*}}(t):=a\left(X_{t}^{\pi^{*}}\right)=\frac{\mu M \gamma}{\delta \sigma^{2}}=\frac{2 \mu M}{\mu^{2}+2 \delta \sigma^{2}}, \quad X_{t}^{\pi^{*}} \geq 0, \\
& l_{\pi^{*}}(t):=l\left(X_{t}^{\pi^{*}}\right)=M, \quad X_{t}^{\pi^{*}} \geq 0 .
\end{aligned}
$$

Moreover, in the case of $M<\mu / 2+\delta \sigma^{2} / \mu$, we have $a\left(X_{t}^{\pi^{*}}\right)<1$ for all $X_{t}^{\pi^{*}} \geq 0$.

In addition, $X_{t}^{\pi^{*}}$ is the solution of

$$
\begin{aligned}
X_{t}^{\pi^{*}}= & X_{0}^{\pi^{*}}+\int_{0}^{t}\left[\mu a_{\pi^{*}}(s)-l_{\pi^{*}}(s)\right] d s \\
& +\int_{0}^{t} \sigma a_{\pi^{*}}(s) d W_{s} .
\end{aligned}
$$

The following theorem shows that the solution $f(x)$ of (14) and (15) constructed above is the optimal value function.

Theorem 7. Let $V_{P}(x)$ be given by (7), and let $f(x)$ be given by (59)-(61) for $M \geq \mu / 2+\delta \sigma^{2} / \mu$ and by (84)-(85) for $M<$ $\mu / 2+\delta \sigma^{2} / \mu$. Then

$$
V_{P}(x)=f(x)
$$

Proof. The proof of this theorem can be carried out in the same way as that of Theorem 2.3 in Højgaard and Taksar [29]. To avoid tedious repetition, we omit it here.

\section{Nonterminal Bankruptcy Model with Bailouts}

4.1. Nonterminal Bankruptcy Model and Its HJB Equation. In the real world, firms may receive financial bailouts from the government or other corporations when they are at the edge of bankruptcy. Therefore, it is necessary for us to consider the optimal dividend problem with capital injections when a firm may encounter bankruptcy. In this section, based on the solutions of (14) and (15), the nonterminal bankruptcy model with bailouts is developed and further its solution is obtained under an appropriate boundary condition.

In order to specify the development of the reserve process in our model when $X_{t}=0$, we consider the following discrete time model of bankruptcy. Assume that at time $t$ the company is at the stage of bankruptcy, and meanwhile the probability it receives an $\epsilon$ amount of external capital inflow is $p h$, while it remains in bankruptcy with probability $1-p h$. Thus, the capital injections of the reserve process at time $t$ are $\Delta_{t}=$ $X_{t+h}-X_{t}$, where $\Delta_{t}(t=0, h, 2 h, \ldots)$ are i.i.d. random variables with the distribution

$$
\Delta_{t}= \begin{cases}0, & \text { with probability } 1-p h \\ \epsilon, & \text { with probability } p h .\end{cases}
$$

Thus,

$$
\mathbb{E} \Delta_{t}=\eta h, \quad \text { where } \eta=p \epsilon .
$$

Again, we adopt the continuous analog

$$
d X_{t}=\eta d t, \quad \text { at } X_{t}=0 .
$$

Combining (4), (5), and (95), we obtain

$$
\begin{aligned}
d X_{t}^{\pi}= & {\left[\mu a_{\pi}(t)-l_{\pi}(t)\right] \mathbf{I}_{\left(X_{t}^{\pi}>0\right)} d t+\eta \mathbf{I}_{\left(X_{t}^{\pi}=0\right)} d t } \\
& +\sigma a_{\pi}(t) \mathbf{I}_{\left(X_{t}^{\pi}>0\right)} d W_{t}, \\
X_{0}^{\pi}= & x .
\end{aligned}
$$

Equation (96) shows that the recovery rate $\eta$ can be viewed as a rate at which the company can receive new capital at the time when $X_{t}^{\pi}=0$. As in Sethi and Taksar [27], for the model considered in this part, we denote it by nonterminal bankruptcy (or bankruptcy with recovery). On the other hand, for the model, which is restricted to stay at the bankruptcy state, it is termed as terminal bankruptcy. Consequently, the optimal value function $V(x)$ becomes as follows:

$$
V(x)=\sup _{\pi \in \Pi} \mathbb{E}_{x}\left[\int_{0}^{\infty} e^{-\delta t} l_{\pi}(t) d t\right] .
$$

Then, the HJB equation satisfied by (98) can be also given by (14). To find the function $V(x)$, we need to specify the behavior of $V(x)$ at the origin. Moreover, its boundary condition should be related to the capital injection rate $\eta$. Now, let us verify the following theorem, which may give us some enlightenment about its boundary condition. 
Theorem 8. Suppose that $J(x)$ is a nonnegative $C^{2}$-function on $[0, \infty)$ with $J^{\prime}(x)$ bounded and $J(x)$ satisfies the HJB equation

$$
\begin{aligned}
& \max _{a \in[0,1], l \in[0, M]}\left[\frac{1}{2} \sigma^{2} a^{2} J^{\prime \prime}(x)+(\mu a-l) J^{\prime}(x)-\delta J(x)\right. \\
& +l]=0, \quad x>0,
\end{aligned}
$$

$$
\begin{aligned}
J(x) & \geq J(x)-\mathbb{E}_{x}\left[e^{-\delta h} J\left(X_{h}^{\pi}\right)\right] \\
= & \mathbb{E}_{x}\left[\int_{0}^{h} e^{-\delta s}\left\{-\left[\frac{1}{2} \sigma^{2} a_{\pi}^{2}(s) J^{\prime \prime}\left(X_{s}^{\pi}\right)+\left(\mu a_{\pi}(s)-l_{\pi}(s)\right) J^{\prime}\left(X_{s}^{\pi}\right)-\delta J\left(X_{h}^{\pi}\right)\right] \mathbf{I}_{\left(X_{s}^{\pi}>0\right)}-\left[\eta J^{\prime}(0)-\delta J(0)\right]\right\} d s\right] \\
+ & \mathbb{E}_{x}\left[\int_{0}^{h} e^{-\delta s} \sigma a_{\pi}(s) J^{\prime}\left(X_{s}^{\pi}\right) \mathbf{I}_{\left(X_{s}^{\pi}>0\right)} d W_{s}\right] .
\end{aligned}
$$

As $J^{\prime}(x)$ is bounded, the last term on the right-hand side is a zero-mean square integrable martingale. Then from (99) and (100), we obtain

$$
J(x) \geq \mathbb{E}_{x}\left[\int_{0}^{h} e^{-\delta s} l_{\pi}(s) \mathbf{I}_{\left(X_{s}^{\pi}>0\right)} d s\right] .
$$

For $X_{s}^{\pi}=0$, it implies that $l_{\pi}(s)=0$. Thus,

$$
\begin{aligned}
& J(x) \\
& \quad \geq \mathbb{E}_{x}\left[\int_{0}^{h} e^{-\delta s}\left\{l_{\pi}(s) \mathbf{I}_{\left(X_{s}^{\pi}>0\right)}+l_{\pi}(s) \mathbf{I}_{\left(X_{s}^{\pi}=0\right)}\right\} d s\right] \\
& \quad=\mathbb{E}_{x}\left[\int_{0}^{h} e^{-\delta s} l_{\pi}(s) d s\right] .
\end{aligned}
$$

Let $h \rightarrow \infty$; using Fatou's lemma and taking the supremum over all policies $\pi \in \Pi$ in (104), we obtain

$$
J(x) \geq V(x), \quad x>0 .
$$

4.2. Solution of the Nonterminal Bankruptcy Model. Although the HJB equation (14) with the mixed boundary condition (100) cannot be solved in a straightforward manner, we can establish a relation between $V(x)$ and $V_{P}(x)$, in which the boundary condition (100) is replaced by (15), by comparing it with the nonzero terminal bankruptcy problem.

Theorem 9. Let $V(x)$ be given by (98). Suppose that $V_{P}(x)$ is the optimal value function for problem (7) and that $\left\{a_{\pi^{*}}(t), l_{\pi^{*}}(t)\right\}$ is the corresponding optimal policy. Suppose

$$
\eta=\frac{\delta V_{P}(0)}{V_{P}^{\prime}(0)}
$$

and let $X_{t}^{\pi^{*}}$ be a solution to the stochastic different equation (96) with $\eta$ given by (106), $a_{\pi^{*}}(t)=a\left(X_{t}^{\pi^{*}}\right)$ and with the mixed Dirichlet-Neumann boundary condition

$$
\eta J^{\prime}(0)-\delta J(0)=0
$$

Then, it follows that

$$
J(x) \geq V(x), \quad x>0 .
$$

Proof. Let $X_{0}^{\pi}=x>0$ and $\pi \in \Pi$ be an admissible policy. For $h>0$, using the generalized Itô's formula, we have
$l_{\pi^{*}}(t)=l\left(X_{t}^{\pi^{*}}\right)$. Then, the optimal value function $V(x)$ can be expressed by $V_{P}(x)$. Moreover, the optimal policy in this model $\pi^{*}$ is given by

$$
\begin{aligned}
& A^{*}(t)=a\left(X_{t}^{\pi^{*}}\right) \mathbf{I}_{\left(X_{t}^{\pi}>0\right)}, \\
& L^{*}(t)=l\left(X_{t}^{\pi^{*}}\right) \mathbf{I}_{\left(X_{t}^{\pi}>0\right)} .
\end{aligned}
$$

Proof. According to Gihman and Skorohod [30], there exists a solution to (96) with $a_{\pi^{*}}(t)=a\left(X_{t}^{\pi^{*}}\right)$ and $l_{\pi^{*}}(t)=l\left(X_{t}^{\pi^{*}}\right)$. Thus, $\left\{A^{*}(t), L^{*}(t)\right\}$ defined by $(107)$ is admissible.

Since $V_{P}(x)$ is a solution of the HJB (14), it follows that

$$
\mathscr{L}^{a, l} V_{P}(x)=0
$$

Equation (106) is equivalent to

$$
\eta V_{P}^{\prime}(0)-\delta V_{P}(0)=0
$$

Repeating the similar argument as the proof of Theorem 8, then by (108) and (109), we obtain

$$
\begin{aligned}
V_{P}(x)-\mathbb{E}_{x}\left[e^{-\delta h} V_{P}\left(X_{h}^{\pi^{*}}\right)\right] \\
\quad=\mathbb{E}_{x}\left[\int_{0}^{h} e^{-\delta s} l_{\pi}\left(X_{s}^{\pi^{*}}\right) \mathbf{I}_{\left(X_{s}^{\pi}>0\right)} d s\right]
\end{aligned}
$$

By boundedness of $V_{P}^{\prime}, V_{P}(y) \leq K(1+y)$ for some $K>0$. Therefore,

$$
e^{-\delta h} V_{P}\left(X_{h}^{\pi^{*}}\right) \leq e^{-\delta h} K\left(1+X_{h}^{\pi^{*}}\right)
$$


Furthermore,

$$
\begin{aligned}
& \mathbb{E}_{x}\left[X_{h}^{\pi^{*}}\right]=\mathbb{E}_{x}[x \\
& +\int_{0}^{h}\left[\mu a\left(X_{s}^{\pi^{*}}\right)-l\left(X_{s}^{\pi^{*}}\right)\right] \mathbf{I}_{\left(X_{s}^{\left.\pi^{*}>0\right)}\right.} d s \\
& \left.+\int_{0}^{h} \eta \mathbf{I}_{\left(X_{s}^{\pi^{*}}=0\right)} d s+\int_{0}^{h} \sigma a\left(X_{s}^{\pi^{*}}\right) \mathbf{I}_{\left(X_{s}^{\pi^{*}}>0\right)} d W_{s}\right] \\
& =\mathbb{E}_{x}\left[x+\int_{0}^{h}\left[\mu a\left(X_{s}^{\pi^{*}}\right)-l\left(X_{s}^{\pi^{*}}\right)\right] \mathbf{I}_{\left(X_{s}^{\pi^{*}}>0\right)} d s\right. \\
& \left.+\int_{0}^{h} \eta \mathbf{I}_{\left(X_{s}^{\pi^{*}}=0\right)} d s\right] \leq x+(\mu+\eta) h .
\end{aligned}
$$

Thus, the term on the left-hand side of (111) is majorized by a positive rand variable with a finite expectation.

Letting $h \rightarrow \infty$ in (110) and using dominated and monotone convergence theorems, we have

$$
V_{P}(x)=V_{\pi^{*}}(x) \leq V(x) .
$$

On the other hand, let $P=V_{P}(0)=\eta V_{P}^{\prime}(0) / \delta$; then according to Theorem 8 , it implies that

$$
V_{P}(x) \geq V(x)
$$

Combining (113) and (114), it shows that $V(x)=V_{P}(x)$.

Next, we will construct an optimal value function $V(x)$ of nonterminal bankruptcy problem with bailouts in the following way. Given a capital injection rate $\eta$, we wish to find a function $P(\cdot)$ depending on $\eta$ such that the function $V_{P(\eta)}(x)$ satisfies

$$
\eta=\frac{\delta V_{P(\eta)}(0)}{V_{P(\eta)}^{\prime}(0)}
$$

Then, according to Theorem 9, we obtain

$$
V(x)=V_{P(\eta)}(x)
$$

For any given $\eta$, a specific analysis of the behavior of $P(\cdot)$ is given as follows.

Case $1\left(M \geq \mu / 2+\delta \sigma^{2} / \mu\right)$. From (59), it is easy to derive that

$$
\eta=\frac{\delta V_{P}(0)}{V_{P}^{\prime}(0)}=\frac{\delta}{\gamma}\left(\frac{P}{C_{1}}\right)^{1 / \gamma},
$$

where $P<C_{1} \beta^{\gamma}$, which implies that $P / C_{1}<\beta^{\gamma}$ and $\eta<$ $\delta \beta / \gamma=\mu / 2$.

In view that $P=0$ implies $\eta=0$, then for the case $\eta \in$ $[0, \mu / 2)$, by (117) we obtain

$$
P=C_{1}\left(\frac{\eta \gamma}{\delta}\right)^{\gamma}
$$

Similarly, from (61), we have

$$
\eta=\frac{\delta V_{P}(0)}{V_{P}^{\prime}(0)}=\frac{\delta P}{(P-M / \delta) \widehat{\theta}}
$$

where $P \geq M / \delta+1 / \widehat{\theta}$. It is easily verified that $\eta(\cdot)$ is a strictly increasing function of $P$, which implies $\eta \geq M+\delta / \widehat{\theta}$.

Thus, for $\eta \in[M+\delta / \widehat{\theta}, \infty)$, (119) implies that

$$
P=\frac{\eta M \hat{\theta}}{\delta(\eta \hat{\theta}-\delta)}
$$

By continuity of $\eta$, for $\eta \in[\mu / 2, M+\delta / \widehat{\theta})$, then applying (60) we get

$$
\eta=\frac{\delta V_{P}(0)}{V_{P}^{\prime}(0)}=\frac{\delta P}{C_{2} \theta_{+}+\left(P-C_{2}\right) \theta_{-}} .
$$

Thus,

$$
P=C_{2} p_{\eta}
$$

where $C_{2}$ is given by (53) and $p_{\eta}=\left(\theta_{+}-\theta_{-}\right) /\left(\delta / \eta-\theta_{-}\right)$.

Substituting (122) into (54) yields that

$$
\begin{aligned}
& \frac{\widehat{\theta}-\theta_{-}}{\theta_{+}\left(\theta_{+}-\theta_{-}\right)} e^{-\theta_{+} x_{1}}+\frac{\theta_{+}-\hat{\theta}}{\theta_{-}\left(\theta_{+}-\theta_{-}\right)} e^{-\theta_{-} x_{1}} \\
& =\frac{\widehat{\theta}-\theta_{-}}{\theta_{+}\left(\theta_{+}-\theta_{-}\right)} e^{-\theta_{+} x_{1}} p_{\eta} .
\end{aligned}
$$

According to (123), $x_{1}$ is given by

$$
x_{1}=\frac{1}{\theta_{+}-\theta_{-}} \ln \left[\frac{\theta_{-}\left(\widehat{\theta}-\theta_{-}\right)}{\theta_{+}\left(\theta_{+}-\hat{\theta}\right)}\left(p_{\eta}-1\right)\right] \text {. }
$$

Combining (53), (122), and (124), it shows that $P$ is uniquely determined by $\eta$.

Remark 10. To ensure that $x_{1}>0$ in (124), we need to verify $\left(\theta_{-}\left(\hat{\theta}-\theta_{-}\right) / \theta_{+}\left(\theta_{+}-\widehat{\theta}\right)\right)\left(p_{\eta}-1\right)>1$ for $\eta \in[\mu / 2, M+\delta / \widehat{\theta})$. Let $h(\eta)=\left(\theta_{-}\left(\hat{\theta}-\theta_{-}\right) / \theta_{+}\left(\theta_{+}-\widehat{\theta}\right)\right)\left(p_{\eta}-1\right)$, then $h(\eta)$ is a strictly decreasing function with respect to $\eta$ on $[\mu / 2, M+$ $\delta / \widehat{\theta})$. Taking $\eta=M+\delta / \widehat{\theta}$ and applying (55), it follows that

$$
\begin{aligned}
& h\left(M+\frac{\delta}{\widehat{\theta}}\right) \\
& =\frac{\theta_{-}\left(\widehat{\theta}-\theta_{-}\right)}{\theta_{+}\left(\theta_{+}-\widehat{\theta}\right)}\left(\frac{\theta_{+}-\theta_{-}}{1 /(M / \delta+1 / \hat{\theta})-\theta_{-}}-1\right) \\
& =\frac{\theta_{-}\left(\widehat{\theta}-\theta_{-}\right)}{\theta_{+}\left(\theta_{+}-\hat{\theta}\right)}\left(\frac{\theta_{+}-\theta_{-}}{\theta_{+} \theta_{-} /\left(\left(\theta_{+}+\theta_{-}\right)-\hat{\theta}\right)-\theta_{-}}-1\right) \\
& =1 .
\end{aligned}
$$

Therefore, $h(\eta)>1$ is surely true for $\eta \in[\mu / 2, M+\delta / \widehat{\theta})$. 
Case $2\left(M<\mu / 2+\delta \sigma^{2} / \mu\right)$. In view of $(84)$, we obtain

$$
\eta=\frac{\delta V_{P}(0)}{V_{P}^{\prime}(0)}=\frac{\delta}{\gamma}\left(\frac{P}{C_{1}}\right)^{1 / \gamma}
$$

where $C_{1}$ is given by (78). The validity of $\eta<M(1-\gamma)$ follows from $P<M(1-\gamma) / \delta$.

Thus, for the case $\eta \in[0, M(1-\gamma))$, by (126) we get

$$
P=C_{1}\left(\frac{\eta \gamma}{\delta}\right)^{\gamma}
$$

On the other hand, for $\eta \in[M(1-\gamma), \infty)$, equivalent to $P \geq M(1-\gamma) / \delta$, (85) implies that

$$
\eta=\frac{\delta V_{P}(0)}{V_{P}^{\prime}(0)}=\frac{\delta M \gamma P}{M-\delta P}
$$

Then,

$$
P=\frac{M \eta}{\delta(M \gamma+\eta)}
$$

Consequently, it shows that for any $\eta \in[0, \infty)$ there exists a unique $P=P(\eta)$, satisfying $\eta=\delta V_{P}(0) / V_{P}^{\prime}(0)$. Then, based on Theorems 5, 6, and 9, we derive the following theorem, which presents an explicit expression for the optimal value function $V(x)$.

Theorem 11. Let $V(x)$ be given by (98).

Case $1\left(M \geq \mu / 2+\delta \sigma^{2} / \mu\right)$. (1) If $\eta \in[0, \mu / 2)$, then $V(x)$ is given by

$$
\begin{aligned}
& V(x) \\
& = \begin{cases}C_{1}\left(x+\frac{\eta \gamma}{\delta}\right)^{\gamma}, & 0 \leq x<x_{0}, \\
C_{1} a_{1} e^{\theta_{+}\left(x-x_{0}\right)}+C_{1} a_{2} e^{\theta_{-}\left(x-x_{0}\right)}, & x_{0} \leq x<x_{1}, \\
\frac{M}{\delta}+\frac{1}{\hat{\theta}} e^{\hat{\theta}\left(x-x_{1}\right)}, & x \geq x_{1},\end{cases}
\end{aligned}
$$

where $x_{0}=(1-\gamma) \sigma^{2} / \mu-\eta \gamma / \delta$ and $x_{1}$ and $C_{1}$ are given by (41) and (42), respectively.

(2) If $\eta \in[\mu / 2, M+\delta / \widehat{\theta})$, then

$$
V(x)= \begin{cases}C_{2} e^{\theta_{+} x}+C_{2}\left(p_{\eta}-1\right) e^{\theta_{-} x}, & 0 \leq x<x_{1}, \\ \frac{M}{\delta}+\frac{1}{\hat{\theta}} e^{\hat{\theta}\left(x-x_{1}\right)}, & x \geq x_{1},\end{cases}
$$

where $x_{1}$ is given by (54) and $C_{2}$ is determined by (53).

(3) If $\eta \in[M+\delta / \widehat{\theta}, \infty)$, then

$$
V(x)=\frac{M}{\delta}+\frac{M}{\eta \hat{\theta}-\delta} e^{\hat{\theta} x}, \quad x \geq 0 .
$$

Case $2\left(M<\mu / 2+\delta \sigma^{2} / \mu\right)$. (1) If $\eta \in[0, M(1-\gamma))$, then $V(x)$ is given by

$V(x)$

$$
= \begin{cases}\frac{1}{\gamma}\left[\frac{M \gamma(1-\gamma)}{\delta}\right]^{1-\gamma}\left(x+\frac{\eta \gamma}{\delta}\right)^{\gamma}, & 0 \leq x<x_{1}, \\ \frac{M}{\delta}\left(1-\gamma e^{-(\delta / M \gamma)\left(x-x_{1}\right)}\right), & x \geq x_{1},\end{cases}
$$

where $x_{1}$ is given by (79).

(2) If $\eta \in[M(1-\gamma), \infty)$, then

$$
V(x)=\frac{M}{\delta}\left(1-\frac{M \gamma}{M \gamma+\eta} e^{-(\delta / M \gamma) x}\right), \quad x \geq 0
$$

Proof. From Theorem 9, (59) and (118), for $\eta \in(0, \mu / 2)$, $V(x)$ can be given by $V(x)=V_{P}(x)$ with $P=C_{1}(\eta \gamma / \delta)^{\gamma}$, that is, (130). By the same method, as the proof of the case $\eta \in(0, \mu / 2)$, we can verify that $V(x)$ is given by (131)-(134), respectively.

4.3. Optimal Policy. According to Section 3.2 and (107), the corresponding admissible policy $\pi^{*}$ for the nonterminal bankruptcy model with bailouts is given as follows.

Case $1\left(M \geq \mu / 2+\delta \sigma^{2} / \mu\right)$. (1) If $\eta \in[0, \mu / 2)$, then

$$
\begin{aligned}
& A^{*}(t) \\
& \quad= \begin{cases}\frac{\mu}{(1-\gamma) \sigma^{2}}\left(X_{t}^{\pi^{*}}+\frac{\eta \gamma}{\delta}\right), & 0 \leq X_{t}^{\pi^{*}}<x_{0}, \\
1, & X_{t}^{\pi^{*}} \geq x_{0},\end{cases}
\end{aligned}
$$

$$
L^{*}(t)= \begin{cases}0, & 0 \leq X_{t}^{\pi^{*}}<x_{1} \\ M, & X_{t}^{\pi^{*}} \geq x_{1}\end{cases}
$$

where $x_{0}=(1-\gamma) \sigma^{2} / \mu-\eta \gamma / \delta$ and $x_{1}$ is given by (41).

(2) If $\eta \in[\mu / 2, M+\delta / \widehat{\theta})$, then

$$
\begin{aligned}
A^{*}(t) & =1, \quad X_{t}^{\pi^{*}} \geq 0, \\
L^{*}(t) & = \begin{cases}0, & 0 \leq X_{t}^{\pi^{*}}<x_{1}, \\
M, & X_{t}^{\pi^{*}} \geq x_{1},\end{cases}
\end{aligned}
$$

where $x_{1}$ is given by (54).

(3) If $\eta \in[M+\delta / \widehat{\theta}, \infty)$, then

$$
\begin{aligned}
A^{*}(t) & =1, \\
L^{*}(t) & =M,
\end{aligned}
$$

$$
X_{t}^{\pi^{*}} \geq 0 .
$$


Case $2\left(M<\mu / 2+\delta \sigma^{2} / \mu\right)$. (1) If $\eta \in[0, M(1-\gamma))$,

$A^{*}(t)$

$$
\begin{aligned}
= & \begin{cases}\frac{\mu}{(1-\gamma) \sigma^{2}}\left(X_{t}^{\pi^{*}}+\frac{\eta \gamma}{\delta}\right), & 0 \leq X_{t}^{\pi^{*}}<x_{1}, \\
\frac{\mu M \gamma}{\delta \sigma^{2}}, & X_{t}^{\pi^{*}} \geq x_{1},\end{cases} \\
L^{*}(t) & = \begin{cases}0, & 0 \leq X_{t}^{\pi^{*}}<x_{1}, \\
M, & X_{t}^{\pi^{*}} \geq x_{1},\end{cases}
\end{aligned}
$$

where $x_{1}$ is given by (79).

(2) If $\eta \in[M(1-\gamma), \infty)$, then

$$
\begin{aligned}
& A^{*}(t)=\frac{\mu M \gamma}{\delta \sigma^{2}}=\frac{2 \mu M}{\mu^{2}+2 \delta \sigma^{2}}, \\
& L^{*}(t)=M,
\end{aligned}
$$

$$
X_{t}^{\pi^{*}} \geq 0
$$

In the case of $M<\mu / 2+\delta \sigma^{2} / \mu$, it is easily seen that $A^{*}(t)<1$ for all $X_{t}^{\pi^{*}} \geq 0$.

In addition, $X_{t}^{\pi^{*}}$ is the solution of

$$
\begin{aligned}
X_{t}^{\pi^{*}}= & X_{0}^{\pi^{*}}+\int_{0}^{h}\left[\mu a_{\pi^{*}}(s)-l_{\pi^{*}}(s)\right] \mathbf{I}_{\left(X_{s}^{\pi^{*}}>0\right)} d s \\
& +\int_{0}^{h} \eta \mathbf{I}_{\left(X_{s}^{\pi^{*}}=0\right)} d s+\int_{0}^{h} \sigma a_{\pi^{*}}(s) \mathbf{I}_{\left(X_{s}^{\pi^{*}}>0\right)} d W_{s} .
\end{aligned}
$$

4.4. Numerical Examples. Based on the calculated parameters $C_{1}, C_{2}, x_{0}$, and $x_{1}$, several numerical examples are provided here to support our theoretical results. It is interesting to look at the relationship between the optimal control and the capital injection rate $\eta$. In Figures 1 and 2, the graphs of the optimal value function $V(x)$ are shown for different $\eta$ under $M=2$ and $M=0.5$, respectively. In these plots, parameters used are given by $\mu=1, \sigma=2$, and $\delta=0.1$. From these graphs, it shows that, for any given $x$, the value of $V(x)$ increases with the capital injection rate $\eta$.

Figures 3 and 4 present the optimal risk exposure $a(x)$ for different $\eta$ at cases $M=2$ and $M=0.5$, respectively. In these graphs, the used parameters are also given by $\mu=1$, $\sigma=2$, and $\delta=0.1$ as in Figures 1 and 2. In the case of $M=2\left(M>\mu / 2+\delta \sigma^{2} / \mu\right)$, for $\eta=0.1<\mu / 2$, the optimal risk exposure $a(x)$ is a linear function of the current reserve process as long as this process is less than $x_{0}=1.78$, after which it is a constant function equal to one. For $\eta=0.6>\mu / 2$, the optimal risk exposure $a(x)$ is constantly equal to one; that is, it is optimal for the company to take the maximum risk all the time and in essence to optimize only the dividend payments. Under the condition $M=0.5\left(M<\mu / 2+\delta \sigma^{2} / \mu\right)$, for $\eta=0.1<M(1-\gamma)$ the optimal risk exposure $a(x)$ monotonically increases from zero to maximum as a linear function of the reserve process and when this value is attained for $x_{1}$ the risk exposure stays here for all $x$ beyond $x_{1}=0.79$. For $\eta=0.3>M(1-\gamma)$, the optimal risk exposure $a(x)$ is

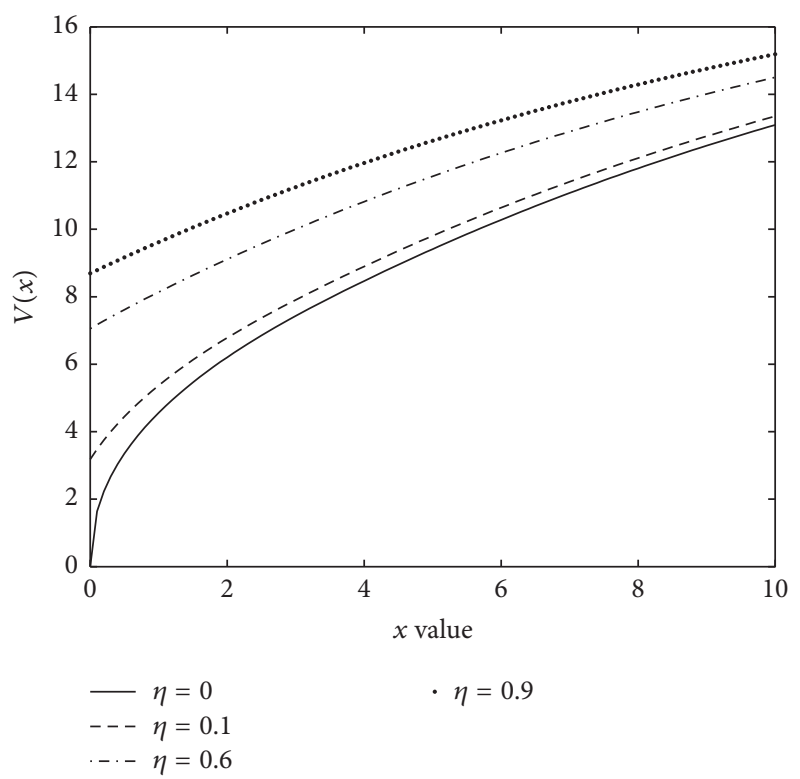

FIgURE 1: The optimal value functions for $\mu=1, \sigma=2, \delta=0.1$, $M=2$ and $\eta=0, \eta=0.1, \eta=0.6, \eta=0.9$.

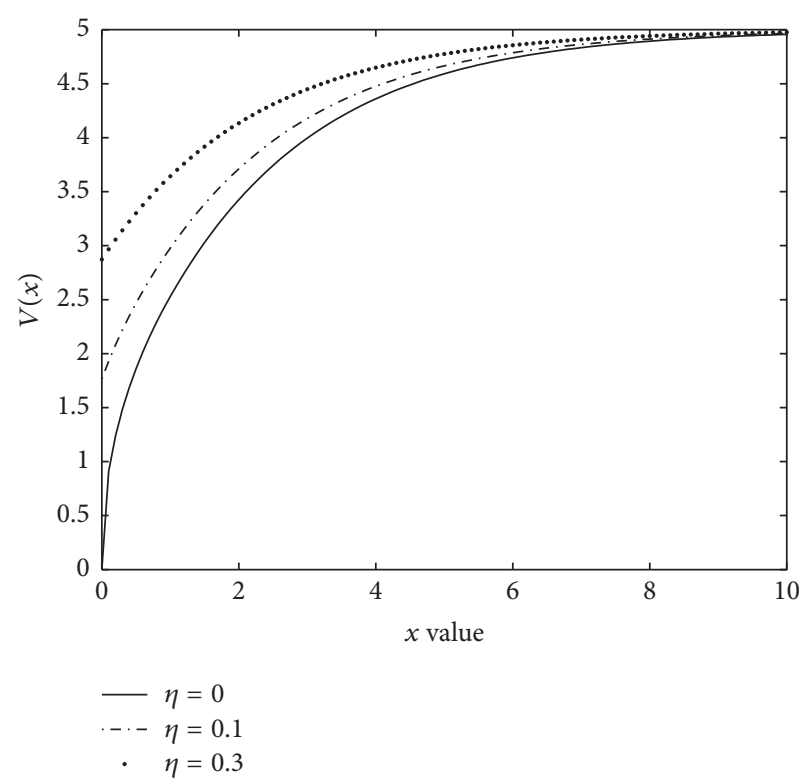

FIgURE 2: The optimal value functions for $\mu=1, \sigma=2, \delta=0.1$, $M=0.5$ and $\eta=0, \eta=0.1, \eta=0.3$.

always equal to the maximum, but less than one. Moreover, it also shows that for any fixed $x$ the optimal risk exposure $a(x)$ is a nondecreasing function of $\eta$.

In Figures 5 and 6 , parameters $\mu, \sigma, \delta$, and $M$ are the same as in Figures 3 and 4, respectively. From the two graphs, it is easily seen that the dividend threshold level $x_{1}$ is a decreasing function with respect to $\eta$.

All of this has the following economic interpretation. In an attempt to pay dividend to the shareholders, at least the company is interested in extending the time before bankruptcy. For an extension of time before bankruptcy, a 


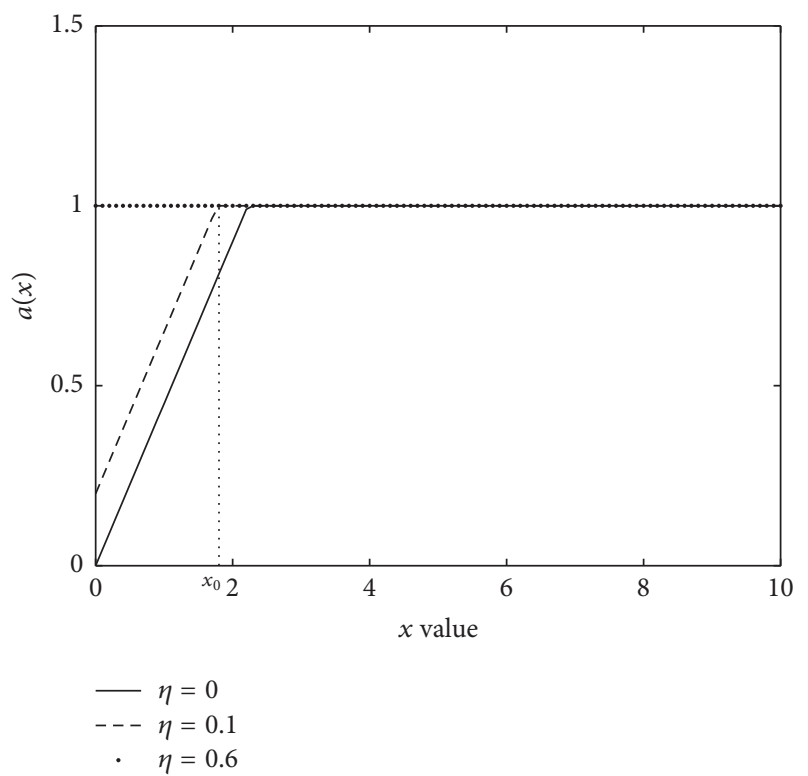

FIGURE 3: The optimal risk exposure for $\mu=1, \sigma=2, \delta=0.1, M=2$ and $\eta=0, \eta=0.1, \eta=0.6$.

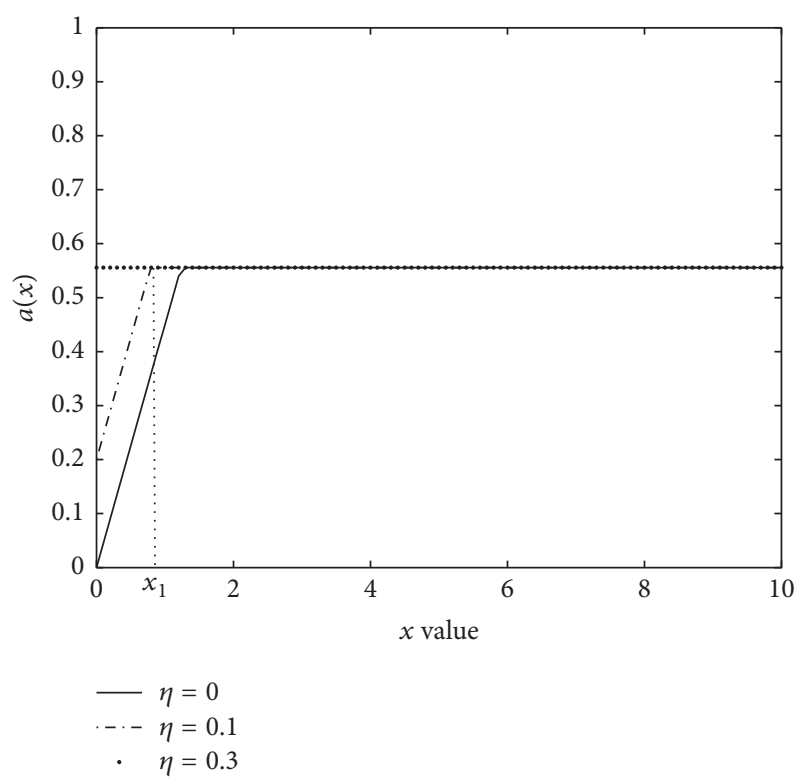

Figure 4: The optimal risk exposure for $\mu=1, \sigma=2, \delta=0.1$, $M=0.5$ and $\eta=0, \eta=0.1, \eta=0.3$.

reduction of risk is needed which simultaneously reduces potential profit. However, with increasing capital injection rate, the company can be less sensitive to bankruptcy, since an increasing rate of capital injection hedges against potential losses of the future profits. The same explanation holds for the decrease of $x_{1}$, when $\eta$ increases. The company can start distributing dividends earlier by lowering the threshold not being concerned that such a policy can bring the bankruptcy faster.

If $M$ is large enough and the reserve or the capital injection rate is large enough, it is optimal for the company

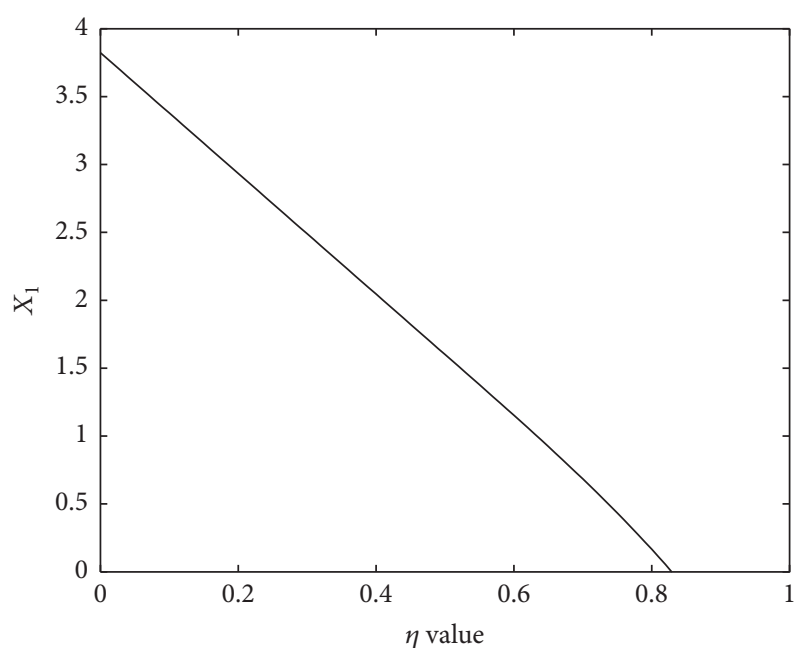

Figure 5: The dividend threshold level $x_{1}$ as a function of $\eta$ with $\mu=1, \sigma=2, \delta=0.1$, and $M=2$.

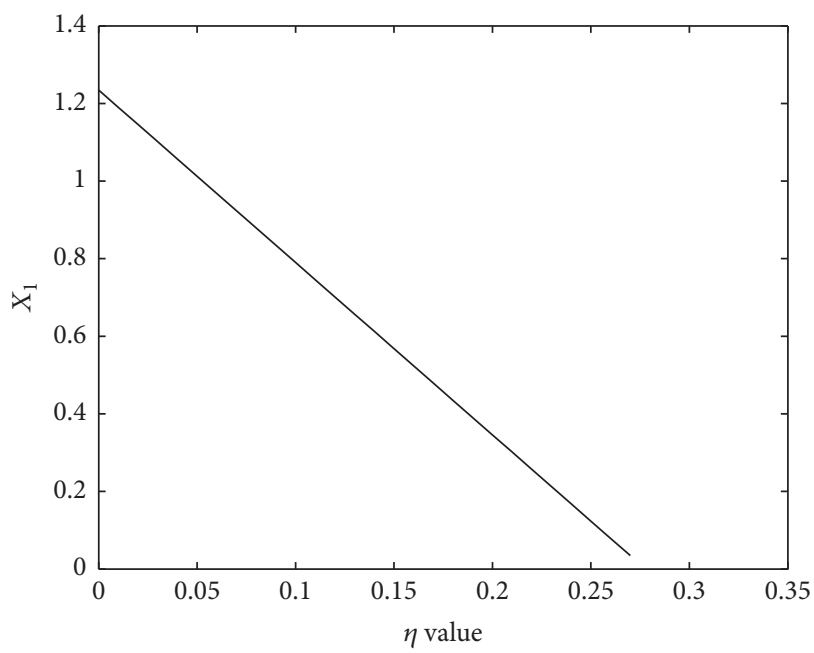

Figure 6: The dividend threshold level $x_{1}$ as a function of $\eta$ with $\mu=1, \sigma=2, \delta=0.1$, and $M=0.5$.

to take the maximal risk exposure all the time. However, if $M$ is small enough, it will never be optimal to take the maximal risk exposure, no matter how large the reserve or the capital injection rate is. In particular, if the rate of capital injection is larger than the upper of the dividend rate, it is the best for a company not to do business at all but to "take money and run."

\section{Conclusions}

In this paper, we develop a stochastic dynamic dividend model with terminal values at bankruptcy status and achieve the analytic solution for the optimal value function. Based on this result, the dividend problem with bailouts at bankruptcy can also be solved and its solution has been constructed. A wonderful result obtained is the concise necessary and sufficient condition for immediate dividend actions after 
bailouts. That is, if the rate of bailouts is greater than the upper of the dividend rate, then the dividend event would happen immediately after bailouts. It demonstrates that to prohibit dividend payout after bailouts the manager or governor should make the rate of bailouts less than the maximum dividend rate.

In the future, following the researches of $\mathrm{Zhu}[31,32]$, Zhu and Zhang [33], Wang and Zhu [34], and so on, we can address some dividend optimization problems for general diffusion models with the drift and diffusion coefficients being general functions of the surplus under the assumption that terminal bankruptcy values are nonzero. For this kind of problem, sometimes we cannot derive the explicit solutions of optimal value function. Then we need to use some suitable numerical methods as in Zhou et al. [35] and Zhou [36] to simulate.

\section{Conflicts of Interest}

The authors declare that there are no conflicts of interest regarding the publication of this paper.

\section{Acknowledgments}

This work was jointly supported by the Scientific Research Fund of Sichuan Provincial Department of Education [Grant no. 11ZA220] and the Funds for "1000 Talents Plan" of Sichuan Province [Grant no. 221410001003040002].

\section{References}

[1] B. de Finetti, "Su un'impostazione alternativa della teoria collettiva del rischio," in Proceedings of the Transactions of the 15th International Congress of Actuaries, vol. 2, pp. 433-443, 1957.

[2] M. Jeanblanc-Picqué and A. N. Shiryaev, "Optimization of the flow of dividends," Russian Mathematical Surveys, vol. 50, no. 2, pp. 257-277, 1995.

[3] R. Radner and L. Shepp, "Risk vs. profit potential: a model for corporate strategy," Journal of Economic Dynamics and Control (JEDC), vol. 20, no. 8, pp. 1373-1393, 1996.

[4] M. I. Taksar and X. Y. Zhou, "Optimal risk and dividend control for a company with a debt liability," Insurance: Mathematics \& Economics, vol. 22, no. 1, pp. 105-122, 1998.

[5] B. Højgaard and M. Taksar, "Optimal proportional reinsurance policies for diffusion models with transaction costs," Insurance: Mathematics \& Economics, vol. 22, no. 1, pp. 41-51, 1998.

[6] B. Højgaard and M. Taksar, "Optimal proportional reinsurance policies for diffusion models," Scandinavian Actuarial Journal, no. 2, pp. 166-180, 1998.

[7] F. Hubalek and W. Schachermayer, "Optimizing expected utility of dividend payments for a Brownian risk process and a peculiar nonlinear ODE," Insurance: Mathematics \& Economics, vol. 34, no. 2, pp. 193-225, 2004.

[8] A. Cadenillas, T. Choulli, M. Taksar, and L. Zhang, "Classical and impulse stochastic control for the optimization of the dividend and risk policies of an insurance firm," Mathematical Finance, vol. 16, no. 1, pp. 181-202, 2006.

[9] J. Paulsen, "Optimal dividend payments until ruin of diffusion processes when payments are subject to both fixed and proportional costs," Advances in Applied Probability, vol. 39, no. 3, pp. 669-689, 2007.

[10] J. Paulsen, "Optimal dividend payments and reinvestments of diffusion processes with both fixed and proportional costs," SIAM Journal on Control and Optimization, vol. 47, no. 5, pp. 2201-2226, 2008.

[11] J. Paulsen and H. K. Gjessing, "Optimal choice of dividend barriers for a risk process with stochastic return on investments," Insurance: Mathematics \& Economics, vol. 20, no. 3, pp. 215-223, 1997.

[12] B. Avanzi and B. Wong, "On a mean reverting dividend strategy with Brownian motion," Insurance: Mathematics \& Economics, vol. 51, no. 2, pp. 229-238, 2012.

[13] M. Hunting and J. Paulsen, "Optimal dividend policies with transaction costs for a class of jump-diffusion processes," Finance and Stochastics, vol. 17, no. 1, pp. 73-106, 2013.

[14] S. Chen, Z. Li, and Y. Zeng, "Optimal dividend strategies with time-inconsistent preferences," Journal of Economic Dynamics \& Control, vol. 46, pp. 150-172, 2014.

[15] J. Eisenberg, "Optimal dividends under a stochastic interest rate," Insurance: Mathematics \& Economics, vol. 65, pp. 259-266, 2015.

[16] M. Vierkötter and H. Schmidli, "On optimal dividends with exponential and linear penalty payments," Insurance: Mathematics \& Economics, vol. 72, pp. 265-270, 2017.

[17] M. Taksar and C. L. Hunderup, "The influence of bankruptcy value on optimal risk control for diffusion models with proportional reinsurance," Insurance: Mathematics \& Economics, vol. 40, no. 2, pp. 311-321, 2007.

[18] M. I. Taksar, "Dependence of the optimal risk control decisions on the terminal value for a financial corporation," Annals of Operations Research, vol. 98, pp. 89-99, 2000.

[19] J. Xu and M. Zhou, "Optimal risk control and dividend distribution policies for a diffusion model with terminal value," Mathematical and Computer Modelling, vol. 56, no. 7-8, pp. 180190, 2012.

[20] P. Chen and B. Li, "Classical and impulse stochastic control on the optimization of dividends with residual capital at bankruptcy," Discrete Dynamics in Nature and Society, vol. 2017, Article ID 2693568, 14 pages, 2017.

[21] N. Kulenko and H. Schimidli, "Optimal dividend strategies in a Cramér-Lundberg model with capital injections," Insurance: Mathematics \& Economics, vol. 43, no. 2, pp. 270-278, 2008.

[22] A. Løkka and M. Zervos, "Optimal dividend and issuance of equity policies in the presence of proportional costs," Insurance: Mathematics \& Economics, vol. 42, no. 3, pp. 954-961, 2008.

[23] L. He and Z. Liang, "Optimal financing and dividend control of the insurance company with proportional reinsurance policy," Insurance: Mathematics \& Economics, vol. 42, no. 3, pp. 976-983, 2008.

[24] L. He and Z. Liang, "Optimal financing and dividend control of the insurance company with fixed and proportional transaction costs," Insurance: Mathematics \& Economics, vol. 44, no. 1, pp. 88-94, 2009.

[25] D. Yao, H. Yang, and R. Wang, "Optimal dividend and capital injection problem in the dual model with proportional and fixed transaction costs," European Journal of Operational Research, vol. 211, no. 3, pp. 568-576, 2011.

[26] Y. Li and G. Liu, "Optimal dividend and capital injection strategies in the Cramér-Lundberg risk model," Mathematical Problems in Engineering, vol. 2015, Article ID 439537, 2015. 
[27] S. Sethi and M. Taksar, "Infinite-horizon investment consumption model with a nonterminal bankruptcy," Journal of Optimization Theory and Applications, vol. 74, no. 2, pp. 333346, 1992.

[28] M. I. Taksar, "Optimal risk and dividend distribution control models for an insurance company," Mathematical Methods of Operations Research, vol. 51, no. 1, pp. 1-42, 2000.

[29] B. Højgaard and M. Taksar, "Controlling risk exposure and dividends payout schemes: Insurance company example," Mathematical Finance, vol. 9, no. 2, pp. 153-182, 1999.

[30] I. I. Gihman and A. V. Skorohod, Stochastic Differential Equations, Springer-Verlag, New York, NY, USA, 1972.

[31] Q. Zhu, "Asymptotic stability in the pth moment for stochastic differential equations with Lévy noise," Journal of Mathematical Analysis and Applications, vol. 416, no. 1, pp. 126-142, 2014.

[32] Q. Zhu, "Razumikhin-type theorem for stochastic functional differential equations with Lévy noise and Markov switching," International Journal of Control, vol. 90, no. 8, pp. 1703-1712, 2017.

[33] Q. Zhu and Q. Zhang, "Pth moment exponential stabilisation of hybrid stochastic differential equations by feedback controls based on discrete-time stable observation with a time delay," IET Control Theory \& Applications, vol. 11, no. 12, pp. 1992-2003, 2017.

[34] B. Wang and Q. Zhu, "Stability analysis of Markov switched stochastic differential equations with both stable and unstable subsystem," Systems \& Control Letters, vol. 105, pp. 55-61, 2017.

[35] Z. Zhou, X. Yu, and N. Yan, "Local discontinuous Galerkin approximation of convection-dominated diffusion optimal control problems with control constraints," Numerical Methods for Partial Differential Equations, vol. 30, no. 1, pp. 339-360, 2014.

[36] Z. Zhou, "A posteriori error estimates for discontinuous Galerkin approximation of non-stationary convection-diffusion optimal control problems," International Journal of Computer Mathematics, vol. 93, no. 12, pp. 2106-2123, 2016. 


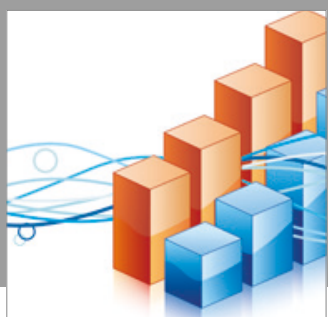

Advances in

Operations Research

vatersals

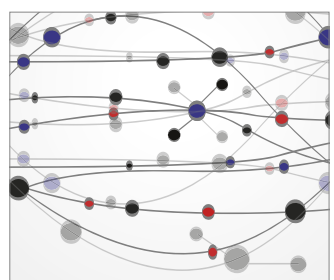

\section{The Scientific} World Journal
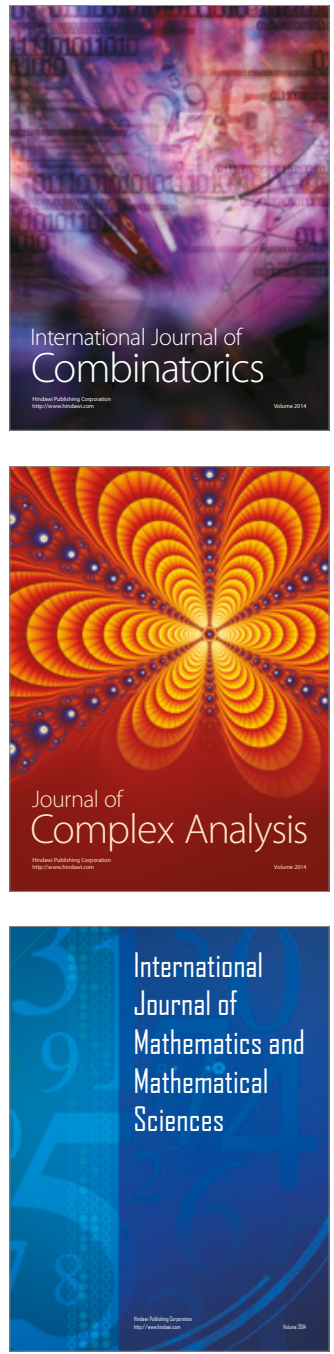
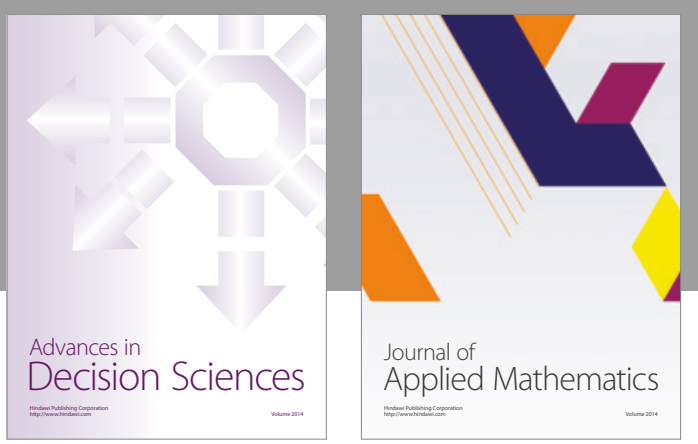

Algebra

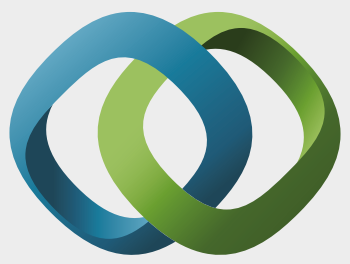

\section{Hindawi}

Submit your manuscripts at

https://www.hindawi.com
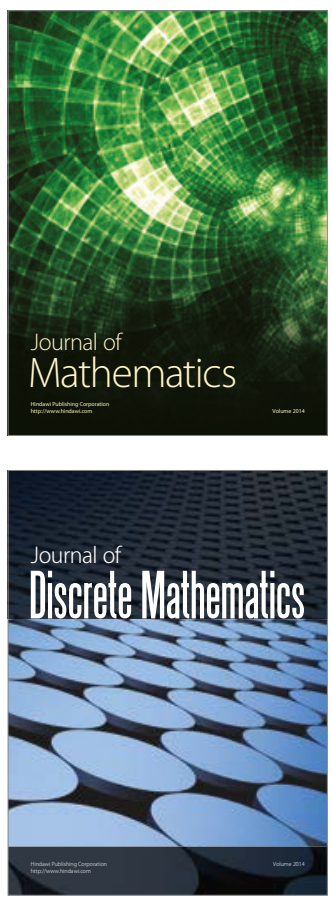

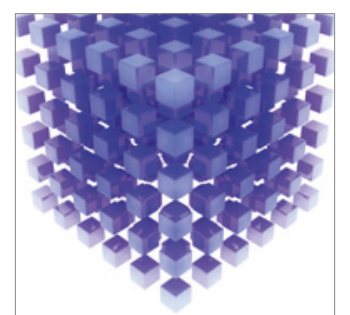

Mathematical Problems in Engineering
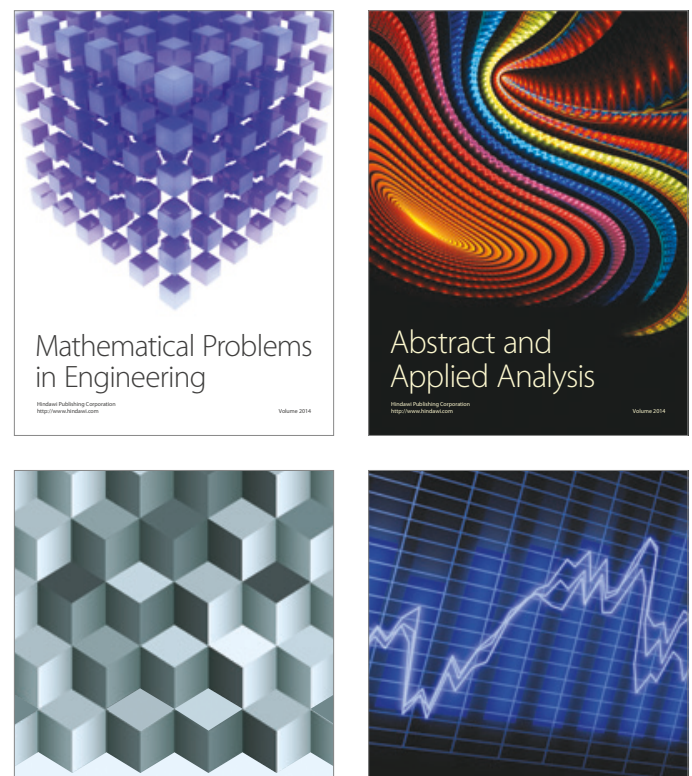

Journal of

Function Spaces

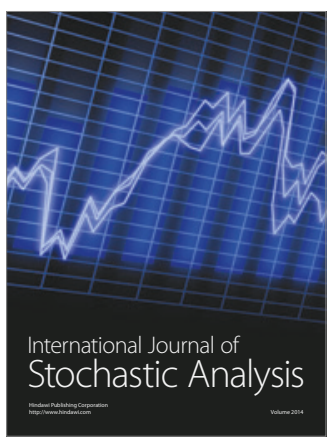

Probability and Statistics
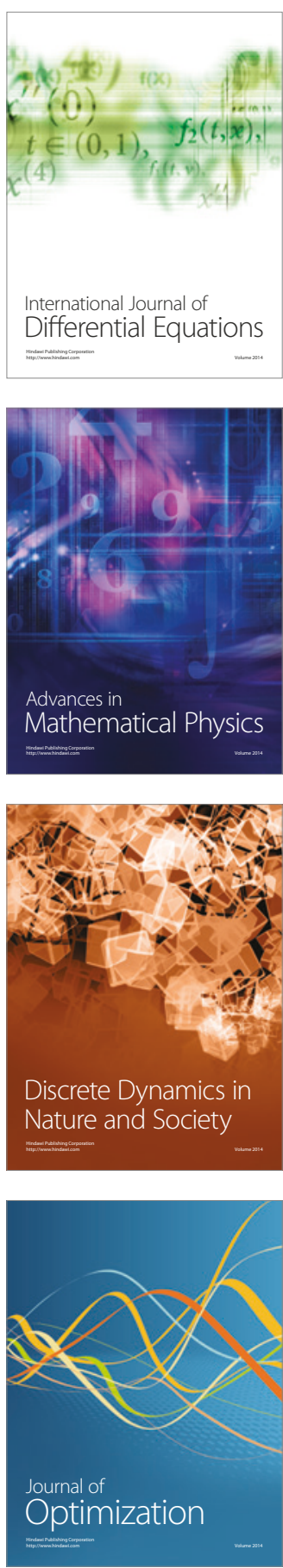